\title{
BMJ Open Long COVID and episodic disability: advancing the conceptualisation, measurement and knowledge of episodic disability among people living with Long COVID - protocol for a mixed-methods study
}

\author{
Kelly K O'Brien (D) , ${ }^{1,2,3,4}$ Darren A Brown (D) , ${ }^{5,6}$ Colm Bergin (D) ,,8 \\ Kristine M Erlandson (D) , Jaime H Vera (D) , ${ }^{10,11}$ Lisa Avery (D) ${ }^{12,13}$ \\ Soo Chan Carusone (D) , ${ }^{14}$ Angela M Cheung (D) ,2,12,15 Susie Goulding, ${ }^{16}$ \\ Richard Harding (D , ${ }^{17}$ Lisa McCorkell, ${ }^{18}$ Margaret O'Hara, ${ }^{19}$ Larry Robinson, ${ }^{20}$ \\ Catherine Thomson, ${ }^{6}$ Hannah Wei, ${ }^{21}$ Natalie St Clair-Sullivan, ${ }^{11}$ Brittany Torres, ${ }^{1}$ \\ Ciaran Bannan, ${ }^{7,8}$ Niamh Roche, ${ }^{22}$ Ruth Stokes, ${ }^{22}$ Patriic Gayle, ${ }^{23}$ \\ Patricia Solomon (1) ${ }^{24}$
}

To cite: O'Brien KK, Brown DA, Bergin C, et al. Long COVID and episodic disability: advancing the conceptualisation measurement and knowledge of episodic disability among people living with Long COVID - protocol for a mixedmethods study. BMJ Open 2022;12:e60826. doi:10.1136/ bmjopen-2022-060826

- Prepublication history and additional supplemental material for this paper are available online. To view these files, please visit the journal online (http://dx.doi.org/10.1136/ bmjopen-2022-060826).

$\mathrm{KKO}$ and $\mathrm{DAB}$ contributed equally.

Received 06 January 2022 Accepted 04 February 2022

Check for updates

(c) Author(s) (or their employer(s)) 2022. Re-use permitted under CC BY-NC. No commercial re-use. See rights and permissions. Published by BMJ.

For numbered affiliations see end of article.

Correspondence to

Dr Kelly K O'Brien;

kelly.obrien@utoronto.ca

\section{ABSTRACT}

Introduction As the prevalence of Long COVID increases, there is a critical need for a comprehensive assessment of disability. Our aims are to: (1) characterise disability experiences among people living with Long COVID in Canada, UK, USA and Ireland; and (2) develop a patientreported outcome measure to assess the presence, severity and episodic nature of disability with Long COVID. Methods and analysis In phase 1, we will conduct semistructured interviews with adults living with Long COVID to explore experiences of disability (dimensions, uncertainty, trajectories, influencing contextual factors) and establish an episodic disability (ED) framework in the context of Long COVID ( $n \sim 10$ each country). Using the conceptual framework, we will establish the Long COVID Episodic Disability Questionnaire (EDQ). In phase 2, we will examine the validity (construct, structural) and reliability (internal consistency, test-retest) of the EDQ for use in Long COVID. We will electronically administer the EDQ and four health status criterion measures with adults living with Long COVID, and readminister the EDQ 1 week later ( $n \sim 170$ each country). We will use Rasch analysis to refine the EDQ, and confirm structural and cross-cultural validity. We will calculate Cronbach's alphas (internal consistency reliability), and intraclass correlation coefficients (testretest reliability), and examine correlations for hypotheses theorising relationships between $\mathrm{EDQ}$ and criterion measure scores (construct validity). Using phase 2 data, we will characterise the profile of disability using structural equation modelling techniques to examine relationships between dimensions of disability and the influence of intrinsic and extrinsic contextual factors. This research involves an academic-clinical-community partnership building on foundational work in ED measurement, Long COVID and rehabilitation.
Strengths and limitations of this study

- Our combined qualitative and quantitative approaches will ensure a comprehensive exploration of experiences among diverse Long COVID populations across healthcare contexts.

- Collaborating with international community networks will provide a robust sampling frame enabling timely recruitment to achieve our sample size, immediate comparisons and rapid uptake of evidence.

- This study draws on a strong conceptual foundation of episodic disability (Episodic Disability Framework) and measurement of disability (Short Form-Episodic Disability Questionnaire) developed using community-engaged approaches with HIV as an exemplar of episodic illness.

- Our cross-sectional nature of the qualitative interviews may limit our ability to document the episodic nature of disability for people living with Long COVID.

- Our ability to attain a representative diverse sample of people with Long COVID given our likelihood to recruit individuals who experience fewer and less severe cognitive and postexertional challenges.

Ethics and dissemination This study was approved by the University of Toronto Research Ethics Board. Knowledge translation will occur with community collaborators in the form of presentations and publications in open access peer-reviewed journals and presentations.

\section{INTRODUCTION}

A growing number of individuals are living with persistent and prolonged signs and 
symptoms following infection consistent with COVID$19,{ }^{12}$ referred to as post-COVID-19 condition or Long COVID. ${ }^{3}{ }^{4}$ Post-COVID-19 condition occurs in children and adults with a history of probable or confirmed SARS CoV-2 infection, usually 3 months from the onset of COVID-19, with symptoms that last for at least 2 months and cannot be explained by an alternative diagnosis. ${ }^{3}$ Symptoms may be new onset following initial recovery from an acute COVID-19 episode or persist from the initial illness. Systematic review evidence examining symptoms among individuals after COVID-19 reported 38\%-72\% were living with $\geq 1$ symptom for at least 2 months from COVID-19 onset ${ }^{5-9}$ and up to $54 \%$ living with symptoms at 6 or more months. ${ }^{9}$ Common symptoms include fatigue, weakness, breathlessness, postexertional symptom exacerbation and cognitive or concentration impairment that can impact daily function ${ }^{23-10}$ and can occur regardless of severity of acute COVID-19. ${ }^{11}{ }^{12}$ Additional challenges with return to employment, relationships, accessing services and stigma, can further contribute to disability after acute COVID-19. ${ }^{13-18}$ Men and women can experience disability differently pertaining to hospitalisation, parental roles, fatigue, mental health and social inclusion. ${ }^{19-22}$ Long COVID may further disproportionately affect people from racialised communities and those with pre-existing comorbidity. ${ }^{22-26}$ With the 'severely disabling, ${ }^{, 16}$ potential of Long COVID, the role for rehabilitation is critical. ${ }^{27-30}$ As such, it is imperative to conceptualise disability in Long COVID and to establish tools to accurately measure disability to inform timely and appropriate access to rehabilitation approaches, and inform policies that consider the complexities of Long COVID. ${ }^{163132}$

Long COVID is multidimensional, episodic and unpredictable, characterised by 'clusters of symptoms' that can overlap and 'fluctuate and change over time', described as 'prolonged', 'relapsing and remitting. ${ }^{3} 17$ '33-38 These characteristics resemble episodic disability (ED), a concept derived from the context of HIV, where health challenges can fluctuate daily or over longer periods of time. ${ }^{39} 40$ There is an opportunity to apply lessons learnt in other chronic and episodic illnesses to understand and conceptualise the disability experienced among adults living with Long COVID.

Members of this team developed the Episodic Disabiltiy (ED) Framework to characterise this multidimensional and sometimes fluctuating nature of health-challenges living with HIV. ${ }^{39}{ }^{41}$ The Episodic Disability Framework includes: (1) dimensions of disability (physical, cognitive, mental and emotional health, daily activities, social inclusion and uncertainty-similar to those reported in Long COVID) ${ }^{23742}$; (2) contextual factors, which include extrinsic (eg, support, environment) and intrinsic (living strategies; personal attributes) factors that influence disability and (3) triggers, that can exacerbate a momentous episode. ${ }^{39} 41$ Empirically validated with adults living with HIV in Canada ${ }^{43}$ and Ireland, ${ }^{44}$ and the first of its kind in the field, the Episodic Disability Framework provides a novel method for conceptualising the multidimensional nature of living with chronic illness by capturing experiences of uncertainty and the temporal (fluctuating) feature of disability over time. ${ }^{43}$ Using this existing framework of disability rigorously developed from others living with episodic illness can provide a foundation for understanding disability experienced by people living with Long COVID. ${ }^{38}$

Standardised patient-reported outcome measures (PROMs) designed to capture the nature and extent of disability and its fluctuation over time are critical to guide the provision of care, determine the effectiveness of interventions, and inform workplace policies, benefits and access to services for people with Long COVID. ${ }^{16}{ }^{17}$ Current disability measures used in Long COVID $^{45}{ }^{46}$ lack items related to uncertainty, ${ }^{33}$ do not capture the fluctuating nature, and were not derived or validated with or from the perspectives of people living with Long COVID. Members of our team developed the HIV Disability Questionnaire (HDQ) a PROM ${ }^{47} 48$ which describes a range of health challenges a person might experience and the extent of fluctuation, so clinicians may better understand and address the disability needs of people with chronic illness. This tool is the first known measure of Episodic Disability, addressing gaps in previously existing health status measures to capture uncertainty and elements of social inclusion (relationships, employment). ${ }^{49}$ The HDQ possesses reliability and validity for use with adults living with HIV in Canada, Ireland, UK, and USA. ${ }^{50-53}$ Recently, our team used modern psychometric (Rasch) and community-engaged approaches to establish a Short-Form version (SF-HDQ) to enhance the feasibility, electronic administration and immediate scoring for use in clinical and community settings. ${ }^{54}$ The lessons learnt in HIV, disability and rehabilitation, specifically our work in Episodic Disability and PROM development provides a foundation for conceptualising and measuring Episodic Disability in the context of Long COVID. ${ }^{33}$

The goal of this study is to advance the conceptualisation and measurement of episodic disability among adults living with Long COVID to inform clinical practice, research and policy. Our specific aims are: (1) to conceptualise the disability experiences (dimensions, trajectories, contextual factors and triggers that exacerbate or alleviate disability over time) among people living with Long COVID in Canada, UK, USA and Ireland, and to establish the Episodic Disability Framework in the context of Long COVID; and (2a) to develop and (2b) assess the measurement properties of an Episodic Disability Questionnaire (EDQ) while (2c) collecting foundational data on the presence, severity and episodic nature of disability and contextual factors (sex, gender, race, age, comorbidity, country) that may influence disability among adults living with Long COVID in Canada, UK, USA and Ireland. 


\section{METHODS AND ANALYSIS}

We will conduct an observational study using mixed methods to establish a conceptual foundation, and PROM, of episodic disability among adults living with Long COVID. We use 'living with Long COVID' throughout to refer to people with Long COVID, regardless of the present nature and severity of symptoms. In the context of this study, 'living with Long COVID' does not assume permanence or recovery, rather recognises the fluctuating, uncertain and potentially unpredictable nature of the course of Long COVID.

\section{Study design}

We will conduct a mixed-methods study using qualitative (aim 1) and quantitative measurement (aim 2) approaches with adults living with Long COVID in Canada, UK, USA and Ireland. Leveraging our work with the Episodic Disability Framework and SF-HDQ, we will examine the cross-cultural applicability and measurement properties of a new electronic (e)-PROM of episodic disability in Long COVID.

\section{Patient and public involvement}

This research involves an international communityacademic-clinical partnership building on foundational work in episodic disability among an international team with expertise in episodic illness, disability, measurement, COVID-19, Long COVID and rehabilitation. This study draws from our work in lessons learnt from HIV for COVID-19 rehabilitation. ${ }^{33} 55$ Building on our longstanding community-engaged research approach, ${ }^{47} 56$ our study involves members of the Long COVID community who were involved in the conceptualisation of the research proposal and acquisition of funding as Collaborators and Knowledge Users. Our team includes people living with Long COVID and representatives from Long COVID community networks (Long COVID Physio, Patient-Led Research Collaborative, Long COVID Support, COVID Long-Haulers Support Group Canada and Long COVID Ireland) ${ }^{57-61}$ who comprise a Community Advisory Committee (CAC) who co-lead and advise on all stages of the research, and coauthor publications and presentations.

\section{Participants and recruitment}

We will recruit individuals who self-identify as having a history of probable or confirmed SARS-CoV-2 infection, and new symptoms occurring within 3 months from the onset of COVID-19 that last for at least 2 months and cannot be explained by an alternative diagnosis. ${ }^{3}$ We will include individuals irrespective of hospitalisation or confirmation of SARS-CoV-2 test (PCR, antigen or antibody). We will recruit adults from Canadian, British, American, Irish and international collaborating Long COVID support networks ${ }^{57-61}$ via email and social media platforms. Community experts/collaborators on the team will lead recruitment as they are best positioned to identify individuals from the community who they identify as representing different characteristics (or combinations of characteristics). We will recruit for diversity in characteristics such as age, sex, gender, race, geographical setting (urban vs rural), employment/student status, socioeconomic status, hospitalisation and country, as able.

\section{Aim 1: interviews}

Community experts will circulate a recruitment poster to individuals in the Long COVID community networks that meet our inclusion and sampling criteria. Interested individuals will contact the respective research coordinator on the team who will send an email to individuals thanking them for interest in the study and attach a consent form for the interview (online supplemental file 1). We will relay information about the purpose and rationale of the study, inclusion criteria, potential of participation, the voluntary nature of the study and their right to withdraw at any time and compensation. On expression of interest, we will send a second email to schedule the one-on-one interview. We will send a third email with additional details, including Zoom information for joining the interview, 1 day prior to the interview.

\section{Aim 2: questionnaire administration}

We will recruit adults living with Long COVID from Canada, UK, USA and Ireland via community networks. Community collaborators will circulate a study poster with contact information for the relevant research coordinator through email or social media. We will apply our lessons learnt using community-engaged approaches with web-based surveys ${ }^{62}$ and a modified Dillman Method ${ }^{63}$ to email potential participants identified via our sampling frame, followed by follow-up reminder emails at 1, 2 and 4 weeks after the initial invitation to facilitate recruitment.

Interested individuals will contact the research coordinator for more information. Research coordinators will send an email that includes the details of the study, with an assigned participant number, and a Qualtrics link to the information cover page (with details of the study, eligibility and consent question) and if eligible/consent, the Time 1 (T1) study questionnaires. The cover page (online supplemental file 2) will include information about the purpose and rationale of the study, inclusion criteria, potential risks, the voluntary nature of the study, and compensation. If an individual participates in the study at T1, we will email a link for the T2 cover page and questionnaire (EDQ only) 1 week later (online supplemental file 3).

\section{Research procedure}

See figure 1 for an overview of the research procedures. Participants will be able to take breaks and schedule the interview or questionnaire completion over more than one session to prevent overexertion resulting postexertional malaise. 
Aim 1 - Conceptualizing disability experiences among people living with Long COVID

Phase 1: Qualitative

40 participants total ( $n=10$ per country)
Aim 2- Developing and assessing the measurement properties of the Episodic Disability Questionnaire (EDQ) with adults living with Long COVID

Phase 2: Measurement

680 participants total ( $n=170$ per country)

Participants: Adults living with Long COVID in Canada, United Kingdom, United States, and Ireland

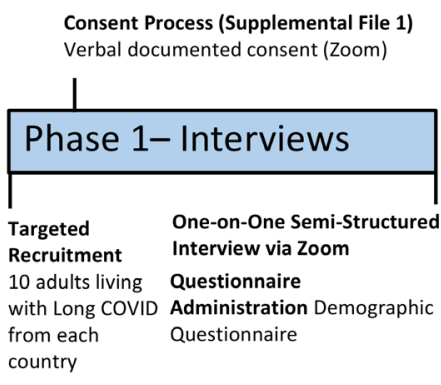

Date Last Revised: February 23, 2022
Consent Process (Supplemental Files 2 \& 3)

Electronic - Embedded within E-questionnaire

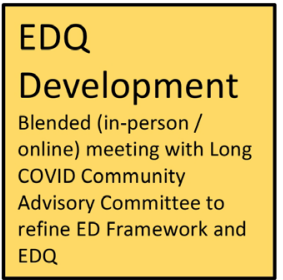

Figure 1 Overview of Research Procedure-Long COVID and Episodic Disability study.

\section{Qualitative data collection (aim 1)}

We will conduct semistructured online (Zoom) interviews with adults who self-identify as living with Long COVID to explore disability experiences and examine the extent to which the Episodic Disability Framework captures their health challenges across our four partner countries. Using an interview guide, we will explore: (1) healthrelated challenges (dimensions of disability: physical, cognitive, mental health, uncertainty, social inclusion, day-to-day activities); (2) trajectories and dimensions of disability experienced as episodic (fluctuating); (3) extrinsic and intrinsic contextual factors that influence disability (eg, comorbid conditions); (4) triggers that may exacerbate an episode and (5) impact (eg, employment, access to services, financial security). We will ask participants to draw their trajectory of health-related challenges (disability) living with Long COVID over time. We will refine the Framework to inform the modification of the EDQ.

\section{Episodic Disability Questionnaire (EDQ) development (aim 2a)}

Using our conceptual framework established in aim 1 we will develop the EDQ, to capture presence, severity and episodic nature of disability. We will host a blended (in-person/online) meeting in consultation with the Long COVID CAC composed of persons living with Long COVID, COVID-19 support networks, researchers and clinicians working in COVID-19 care to refine the Episodic Disability Framework and EDQ in preparation for implementation. Episodic Disability Questionnaire (EDQ) items will be derived from categories in the Framework (aim 1), adapted from the original SF-HDQ, and refined with the CAC.

\section{Quantitative data collection (aim $2 \mathrm{~b}$ and $2 \mathrm{c}$ )}

We will electronically administer the Episodic Disability Questionnaire (EDQ) followed by four criterion measures currently used in Long COVID: WHO Disability
Assessment Schedule, ${ }^{45}$ COVID-19 Yorkshire Rehabilitation Scale (C19-YRS) ${ }^{46}$; EuroQol- EQ-5D-5L quality of life questionnaire, ${ }^{64}$ Work and Social Adjustment Scale (WSAS) ${ }^{65}$ using e-survey software (Qualtrics) at Time $1 .{ }^{66}$ The EDQ (only) will be readministered electronically a second time, 1 week later (time 2). At time 2, we will ask participants whether they had any major changes in their health since Time 1 (to indicate consistency in disability for reliability assessment of the EDQ) (figure 1).

\section{WHO Disability Assessment Schedule 2.0 (WHODAS 2.0)}

The WHODAS 2.0 contains 36 items that measures disability across six domains (cognition, mobility, selfcare, getting along, life activities and participation). Scores ranges from 0 to 100 , where 0 means no disability and 100 means full disability. The WHODAS 2.0 possesses reliability, validity and responsiveness among people with chronic disease. ${ }^{45}$

\section{COVID-19 Yorkshire Rehabilitation Scale}

The C19-YRS is a digital assessment and monitoring tool that screens for some of the most common symptoms of Long COVID, including breathlessness, cough, fatigue, depression and anxiety. ${ }^{467}$ The scale consists of 22 items with each item scored on an 11-point numerical rating scale from 0 (none of this symptom) to 10 (extremely severe level or impact). The C19-YRS is divided into four subscales (range of total score for each subscale): symptom severity score (0-100), functional disability score $(0-50)$, additional symptoms $(0-60)$ and overall health $(0-10)$. The C19-YRS possesses internal consistency, content validity and clinical usefulness for use with persons with Long COVID. ${ }^{67}$

\section{EuroQol EQ-5D-5L}

The EQ-5D-5L is a measure of health-related quality of life widely used in cost effectiveness analysis. The EQ-5D-5L consists of 5 items (mobility, self-care, usual activities, 
pain/discomfort, anxiety/depression) each with 5 levels of response options, followed by a visual analogue scale (overall health) ${ }^{64}$ and has been utilized as a measure of quality of life in the context of COVID-19. ${ }^{1368}$

\section{Work and Social Adjustment Scale}

The WSAS is a five-item questionnaire asking about the impact of a health condition on ability to work, home management, social leisure activities, private leisure activities and relationships with others. ${ }^{65}$ The WSAS has demonstrated utility, reliability and validity as a measure of functional impairment for use with people with mental health conditions.

\section{Demographic questionnaire}

In aims 1 and 2 (time 1 only), we will administer a demographic questionnaire capturing factors such as: age, sex, gender, sexual orientation, race, country, employment status, multimorbidity, access to supports, living situation (alone vs not), COVID-19 vaccination status, severity of acute COVID-19 (non-hospitalised, hospitalised versus Intensive Care Unit (ICU)), timing of acute COVID19, Long COVID symptom onset, and access to a Long COVID clinic.

\section{Analysis}

Interview data (aim 1)

We will analyse interviews using content analytical techniques $^{69}$ and a team based approach to qualitative analysis involving community collaborators. ${ }^{70} 71$ Our coding scheme will be informed by the Episodic Disability Framework and include: (1) dimensions of health challenges; (2) factors that trigger, exacerbate or alleviate disability; (3) episodic nature of disability (trajectories); (4) uncertainty living with Long COVID and (5) contextual considerations (eg, country, gender). We will also allow additional codes to emerge to identify new features of disability with Long COVID. We will use NVivo software for data management. ${ }^{72}$

\section{Episodic Disability Questionnaire (EDQ) development (aim 2a)}

We will conduct a content analysis on the discussion notes taken from our consultation meeting with the Long COVID CAC to refine the Episodic Disability Framework (aim1) and Episodic Disability Questionnaire (EDQ).

\section{Questionnaire data}

We will calculate EDQ disability presence, severity and episodic (calculated for descriptive purposes only) across the six domains and total scores (transformed out of 100) for each time point. Higher scores indicate a higher presence, severity and episodic nature of disability. WHODAS 2.0 scores ranges from 0 to 100 , where 0 means no disability and 100 means full disability. We will calculate mean (SD) and median (IQR) domain scores. The C19YRS is divided into four subscales (range of total score for each subscale): symptom severity score $(0-100)$, functional disability score $(0-50)$, additional symptoms $(0-60)$ and overall health $(0-10)$. For the EQ5D-5L divide the dimensions into five levels of health problems to collectively represent a health state that represent each of the numerical descriptions. ${ }^{64}$ For the WSAS, we will calculate a total score. A score above 20 suggests moderately severe or worse psychopathology, scores between 10 and 20 are associated with significant functional impairment but less severe clinical symptomatology, and scores below 10 are associated with subclinical populations. Individual severity scores are derived by summing the values (scores) for the six questions for each individual. ${ }^{65}$ For the demographic questionnaire, we will calculate descriptive statistics including frequencies (\%) for categorical variables and median and IQR for continuous variables.

\section{Property assessment (aim 2b)}

We will conduct Rasch analysis, a preferred method for developing PROMs, assessing structural and cross-cultural validity, and for establishing an interval-level scale. ${ }^{73-76}$ We will use criteria for model fit similarly used in our SF-HDQ development ${ }^{54}$ : Cronbach's alpha (internal consistency reliability $(\geq 0.8$ acceptable $),{ }^{77}$ and Person Separation Indices $(\geq 0.70$ acceptable) $){ }^{78}$ individual item fit using item threshold ordering, fit residuals (residuals $>|2.5|),{ }^{79} 80$ differential item functioning (country, age, sex, gender $)^{74}$ and unidimensionality. ${ }^{81}$ We will create a user-friendly scoring algorithm to convert raw summed EDQ scores to the equivalent Rasch-based person logit scores rescaled (0-100) with higher scores indicating greater severity of disability. ${ }^{82}$

We will assess test-retest reliability with ICC using time 1 and 2 EDQ scores ( $>0.8$ acceptable) ${ }^{77}{ }^{83}$ We will examine correlations for a priori hypotheses theorising relationships between $\mathrm{EDQ}$ and criterion measure scores. Correlations of $|\geq 0.30|,|\geq 0.50|$ and $|\geq 0.70|$ will be defined as 'weak', 'moderate' and 'strong,' respectively. ${ }^{84}$ Construct validity will be defined as $>75 \%$ confirmed hypotheses. ${ }^{85}$

\section{Disability profile (aim 2c)}

We will use descriptive and structural equation modelling techniques to examine relationships between dimensions of disability and contextual factors. ${ }^{86} 87$ We will conduct path analyses to determine relationships between dimensions of disability and contextual factors (sex, gender, age, race, multimorbidity, hospitalisation, country, acute disease severity, symptom clusters, vaccination status) in a stepwise fashion to determine how these factors influence disability. We will estimate parameter values using Maximum Likelihood Methods of Estimation. ${ }^{88}$ We will assess overall goodness of fit (Root Mean Square Error of Approximation $(<0.05)$, Comparative Fit Indices $(\geq 0.95)$, Tucker Lewis Index $(\geq 0.95))$ and the magnitude and direction of the parameter estimates to determine overall fit of our model.$^{89-91}$ We will use SAS V $9.3,{ }^{92}$ RUMM $2030^{93}$ and Mplus statistical software ${ }^{94}$ to facilitate analysis and discuss model interpretations and implications with the team. 


\section{Sample size}

For aim 1, based on our previous work cross-cultural assessment of the Episodic Disability Framework, ${ }^{51}$ we anticipate a sample of 40 across 4 countries ( 10/ country) will be sufficient to provide perspectives of disability internationally. ${ }^{95}$ For aim 2, our sample size is based on the Rasch analysis (aim 2b). To maximise item parameter estimation precision with minimal root mean squared deviation, we require at least 500 observations. ${ }^{96}$ To account for missing responses $(\sim 35 \%)$ at time 2 (testretest), we will collect data on 680 participants (170 per country).

\section{DISCUSSION}

Results will yield the first known conceptual framework and PROM developed to assess the prevalence and impact of episodic disability in Long COVID. Specific outcomes will include: a conceptual framework (and definition) of Episodic Disability in the context of Long COVID; foundational knowledge on the profile and impact of episodic disability within Canadian, UK, USA and Irish Long COVID health contexts; a new e-PROM of episodic disability assessed for cross-cultural validity and reliability, poised for broad clinical and research use to enable future international comparisons; a guidance document for EDQ utility, administration and scoring; and a new and strengthened multidisciplinary and international team of clinicians, researchers, and community members dedicated to addressing research priorities in Long COVID and rehabilitation.

Our combined qualitative and quantitative approaches will ensure a robust exploration of diverse experiences across Long COVID populations and healthcare contexts. Results can inform strategies for targeting interventions, and policies for return to work, health and income benefits for people living with Long COVID. Discerning crosscultural applicability of the ED Framework and EDQ will help to establish a common understanding of disability with Long COVID to facilitate future research and international comparisons. Collaborators, Long COVID Support (UK), ${ }^{58}$ COVID Long-Haulers Support Group Canada, ${ }^{57}$ Patient-Led Research Collaborative (US), ${ }^{60}$ Long COVID Physio, ${ }^{59}$ Long COVID Ireland $^{61}$ and Canada-International HIV and Rehabilitation Research Collaborative $^{9798}$ will further leverage integrated knowledge translation.

This study addresses priorities outlined by the Long COVID community ${ }^{99}$ and COVID-19 Rehabilitation Research Framework ${ }^{100}$ highlighting the need to measure disability to inform best practices and policies for rehabilitation. The framework and Episodic Disability Questionnaire (EDQ) that emerges from this work may yield potential to lay the groundwork for future disability measurement in Long COVID and more broadly across other complex episodic illness. ${ }^{55}$

\section{ETHICS AND DISSEMINATION}

This protocol was approved by the University of Toronto Health Sciences Research Ethics Board (Protocol Reference \#41749) on 17 November 2021.

\section{Consent}

Phase 1 (qualitative interviews)

Interested individuals will contact the coordinator in each country who will email them an overview of the study with the information/consent form (online supplemental file 1). The individual will be informed of the purpose and rationale of the study, inclusion criteria, potential medical and social risks of participation, the voluntary nature of the study and their right to withdraw at any time, and compensation. Verbal consent for the interview will be taken by the research investigator (over Zoom) at the time of the scheduled interview. The research team member will sign and date the consent form, confirming that verbal consent was obtained.

\section{Phase 2 (questionnaire administration)}

Research coordinators will send an initial recruitment email that includes the details of the study, a Qualtrics link to the information cover page. If individuals indicate they are eligible and consent to participate (in Qualtrics), they will be brought to the time 1 (T1) study questionnaires. If an individual participates in the study at T1 (consents to participate and completes the T1 questionnaires), we will email a link for the T2 questionnaire (EDQ only) 1 week later. T1 and T2 cover pages include an electronic eligibility/consent form for participants to complete prior to proceeding with the questionnaire(s). Consent will also be implied with completion of the questionnaires (online supplemental file 2) (T1 cover page) and (online supplemental file 3) (T2 cover page).

\section{Token of appreciation}

Participants living in Canada, USA, UK and Ireland will be provided with a $\$ \mathrm{C} 30$, US $\$ 20, £ 15$ gift card and $€ 20$, respectively, as a token of appreciation for participation in the interview and completion of the demographic questionnaire (phase 1). We will provide participants with a similar token of appreciation for completing both time 1 and 2 questionnaires (phase 2).

\section{Potential risks and benefits}

Some adults living with Long COVID may find the interview or questionnaire completion to be cognitively demanding. Participants will be able to take breaks and schedule the interview or questionnaire completion over more than one session. Some of the questions on the questionnaires or in the interviews may be personal or sensitive in nature. Participants can choose not to answer questions and may end the interview or close their browser with the questionnaires on Qualtrics at any time. Interviewers will send participants a follow-up check-in email with contact information for the principal investigator (country lead) and for supportive services at specific community collaborator networks. 
There are no direct benefits to participants taking part in this study. However, it may help to develop a new measure of disability and advance knowledge about the disability that adults living with Long COVID experience.

\section{Confidentiality \& Data Management}

All participant records will be identified by a coded number to maintain confidentiality. A master list of participants with their respective participant numeric codes and contact information will be stored on a passwordprotected computer file by the investigator in the respective country. The master file linking participant names and email addresses to their number will remain in their country of origin. Electronic copes of the phase 1 (interview) consent forms will be stored on a secure server at the University of Toronto (Canada).

On completion of the interviews, we will immediately upload audio files to Sharefile, a password-protected secure sharing and encrypted transfer system. ${ }^{101}$ Recordings will be deleted from the recording device and identification numbers will replace participant identifiers on corresponding data.

All questionnaire responses will be downloaded from Qualtrics, an online secure e-survey software that uses Transport Layer Security encryption, ${ }^{66}$ and transferred to the University of Toronto using Sharefile, ${ }^{101}$ and stored on a secure server at the University of Toronto. Anonymised data will be shared among investigators using Sharefile. Electronic data will be stored on a password-protected computer in a locked office at the University of Toronto and will be accessible only to investigators and research coordinators. Data will be anonymised when compiling and disseminating results. An anonymised aggregated dataset for this study will be made open access as per the funding conditions of the Canadian Institutes of Health Research via the University of Toronto.

Following the completion of the study, printed and electronic data (excluding audio recorded data) will be retained for 10 years in the same location that it was stored throughout the study. Audio files will be destroyed immediately following publication. Files containing personal contact information will be deleted after publication. All remaining electronic and hard copy data and information related to the study will be retained for 10 years after the completion of the study and then destroyed by the principal investigator (Toronto).

\section{Dissemination}

We will translate results among people living with Long COVID, researchers, clinicians and community organisations. Integrated knowledge translation will be led by the community advisory community and facilitated by community networks and organisations. We will develop an Episodic Disability and Long COVID Toolkit, shared widely with researchers, community groups and clinicians that will include approaches for conceptualising disability for people living with Long COVID. Further knowledge translation will occur via publications in open access peerreviewed scientific journals, presentations at academic conferences, community organisations, webinars and education of trainees, in partnership with community collaborators.

\section{Author affiliations}

${ }^{1}$ Department of Physical Therapy, Temerty Faculty of Medicine, University of Toronto, Toronto, Ontario, Canada

${ }^{2}$ Institute of Health Policy, Management and Evaluation (IHPME), Dalla Lana School of Public Health, University of Toronto, Toronto, Ontario, Canada

${ }^{3}$ Rehabiltation Sciences Institute (RSI), University of Toronto, Toronto, Ontario, Canada

${ }^{4}$ Long COVID Physio, Toronto, Ontario, Canada

${ }^{5}$ Therapies Department, Chelsea and Westminster Hospital NHS Foundation Trust, London, UK

${ }^{6}$ Long COVID Physio, London, UK

${ }^{7}$ Department of Genitourinary and Infectious Diseases (GUIDE Clinic), St. James's Hospital, Dublin, Ireland

${ }^{8}$ Trinity College Dublin, School of Medicine, Dublin, Ireland

${ }^{9}$ University of Colorado Anschutz Medical Campus, Aurora, Colorado, USA

${ }^{10}$ Brighton and Sussex Medical School, University of Sussex Brighton, Brighton, UK

${ }^{11}$ Royal Sussex County Hospital, Brighton and Sussex University Hospitals NHS

Foundation Trust, Brighton, UK

${ }^{12}$ University Health Network, Toronto, Ontario, Canada

${ }^{13}$ Dalla Lana School of Public Health, University of Toronto, Toronto, Ontario, Canada

${ }^{14}$ McMaster Collaborative for Health and Aging, McMaster University, Hamilton, Ontario, Canada

${ }^{15}$ Department of Medicine, University of Toronto, Toronto, Ontario, Canada

${ }^{16}$ COVID Long-Haulers Support Group Canada, Toronto, Ontario, Canada

${ }^{17}$ Cicely Saunders Institute of Palliative Care, Policy \& Rehabilitation, King's College

London, London, UK

${ }^{18}$ Patient-Led Research Collaborative, Washington, DC, USA

${ }^{19}$ Long COVID Support, Birmingham, UK

${ }^{20}$ Sunnybrook Health Sciences Centre, Sunnybrook Research Institute, Toronto, Ontario, Canada

${ }^{21}$ Patient-Led Research Collaborative, Ottawa, Ontario, Canada

${ }^{22}$ Long COVID Ireland, Dublin, Ireland

${ }^{23}$ Canada-International HIV and Rehabilitation Research Collaborative (CIHRRC), London, UK

${ }^{24}$ School of Rehabilitation Science, McMaster University Faculty of Health Sciences, Hamilton, Ontario, Canada

Correction notice This article has been corrected since it was first published. The figure has been updated.

Twitter Kelly K 0'Brien @kellyobrien25, Darren A Brown @darrenabrown, Kristine M Erlandson @kerlands, Richard Harding @RHardingCSI and Ruth Stokes @ longcovidirl

Acknowledgements We thank Hannah Davis, Patient-Led Research Collaborative (PLRC) for their role in fostering collaborations with the Long COVID community, the development and refinement of the research protocol and acquisition of funding.

Contributors KKO'B and DAB co-led the conceptualisation of the study objectives, and drafted the protocol, lead the application for acquisition of funding and are the co-lead investigators on the study. KKO'B, DAB, JV, CBe and KME, JV are coprincipal investigators and country leads on the research team, and were involved in the conceptualisation of the study design, development of the protocol and acquisition of funding. LA, SCC, AC, LR, RH and PS are coinvestigators and were involved in the conceptualisation of the study design and contributed to the development of the protocol, and acquisition of funding. SG, LM, MO'H, CT, HW, RS and NR are collaborators and community experts in the Long COVID community who were involved in the review and refinement of the protocol. They are members of the Community Advisory Committee and will lead recruitment of participants and community-integrated knowledge translation. PG is a community collaborator and knowledge translation expert who will be involved in the knowledge translation. NSC-S and BT are research coordinators involved in the recruitment and data collection and contributed to the refinement of the protocol. CBa is a clinician working in Long COVID and coinvestigator who recently joined the team and 
who reviewed of the protocol. All authors read and approved the final protocol manuscript.

Funding This work is supported by the Canadian Institutes of Health Research (CIHR), Emerging COVID-19 Research Gaps and Priorities Funding Opportunity (Funding Research Number \#: GA4-177753), 160 Elgin Street, Ottawa, Ontario, Canada, K1A OW9). KKO'B is supported by a Tier 2 Canada Research Chair in Episodic Disability and Rehabilitation and AC is supported by a Tier 1 Canada Research Chair in Musculoskeletal and Postmenopausal Health from the Canada Research Chairs Programme.

Competing interests None declared.

Patient consent for publication Not applicable.

Provenance and peer review Not commissioned; peer reviewed for ethical and funding approval prior to submission.

Supplemental material This content has been supplied by the author(s). It has not been vetted by BMJ Publishing Group Limited (BMJ) and may not have been peer-reviewed. Any opinions or recommendations discussed are solely those of the author(s) and are not endorsed by BMJ. BMJ disclaims all liability and responsibility arising from any reliance placed on the content. Where the content includes any translated material, BMJ does not warrant the accuracy and reliability of the translations (including but not limited to local regulations, clinical guidelines, terminology, drug names and drug dosages), and is not responsible for any error and/or omissions arising from translation and adaptation or otherwise.

Open access This is an open access article distributed in accordance with the Creative Commons Attribution Non Commercial (CC BY-NC 4.0) license, which permits others to distribute, remix, adapt, build upon this work non-commercially, and license their derivative works on different terms, provided the original work is properly cited, appropriate credit is given, any changes made indicated, and the use is non-commercial. See: http://creativecommons.org/licenses/by-nc/4.0/.

\section{ORCID iDs}

Kelly K O'Brien http://orcid.org/0000-0002-1632-6537

Darren A Brown http://orcid.org/0000-0002-4956-243X

Colm Bergin http://orcid.org/0000-0002-6651-1132

Kristine M Erlandson http://orcid.org/0000-0003-0808-6729

Jaime H Vera http://orcid.org/0000-0002-1165-0573

Lisa Avery http://orcid.org/0000-0002-8431-5143

Soo Chan Carusone http://orcid.org/0000-0003-3977-0523

Angela M Cheung http://orcid.org/0000-0001-8332-0744

Richard Harding http://orcid.org/0000-0001-9653-8689

Patricia Solomon http://orcid.org/0000-0002-5014-0795

\section{REFERENCES}

1 National Institute for Health and Care Excellence (NICE). COVID-19 rapid guideline: managing the long-term effects of COVID-19, 2020. Available: https://www.nice.org.uk/guidance/ng188

2 Nalbandian A, Sehgal K, Gupta A, et al. Post-acute COVID-19 syndrome. Nat Med 2021;27:601-15.

3 Soriano JB, Murthy S, Marshall JC, et al. A clinical case definition of post-COVID-19 condition by a Delphi consensus. Lancet Infect Dis 2021. doi:10.1016/S1473-3099(21)00703-9. [Epub ahead of print: 21 Dec 2021].

4 Callard F, Perego E. How and why patients made long Covid. Soc Sci Med 2021;268:113426.

5 Nasserie T, Hittle M, Goodman SN. Assessment of the frequency and variety of persistent symptoms among patients with COVID-19. a systematic review. JAMA Netw Open 2021;4:e2111417.

6 Domingo FR, Waddell LA, Cheung AM. Prevalence of long-term effects in individuals diagnosed with COVID-19: a living systematic review. medRxiv 2021

7 Michelen M, Manoharan L, Elkheir N, et al. Characterising long COVID: a living systematic review. BMJ Glob Health 2021;6.

8 Whitaker M, Elliott J, Chadeau-Hyam M. Persistent symptoms following SARS-CoV-2 infection in a random community sample of 508,707 people. medRxiv 2021.

9 Groff D, Sun A, Ssentongo AE, et al. Short-term and long-term rates of Postacute sequelae of SARS-CoV-2 infection: a systematic review. JAMA Netw Open 2021;4:e2128568.

10 Taquet M, Dercon Q, Luciano S, et al. Incidence, co-occurrence, and evolution of long-COVID features: a 6-month retrospective cohort study of 273,618 survivors of COVID-19. PLoS Med 2021;18:e1003773.
11 Estiri H, Strasser ZH, Brat GA, et al. Evolving phenotypes of non-hospitalized patients that indicate long COVID. BMC Med 2021;19:249.

12 Blomberg B, Mohn KG-I, Brokstad KA, et al. Long COVID in a prospective cohort of home-isolated patients. Nat Med 2021;27:1607-13.

13 Tabacof L, Tosto-Mancuso J, Wood J, et al. Post-acute COVID-19 syndrome negatively impacts physical function, cognitive function, health-related quality of life, and participation. Am J Phys Med Rehabil 2022;101:48-52.

14 National Institute for Health Research (NIHR). Living with COVID19: second review, 2021. Available: https://evidence.nihr.ac.uk/ themedreview/living-with-covid19-second-review/

15 Brown D, Oller D, Hassell H. Physical therapists living with long COVID, part 1: defining the Indefinable. JOSPT 2021 https://www. jospt.org/do/

16 Rajan S, Khunti K, Alwan N. In the wake of the pandemic: preparing for long COVID: policy brief 39, 2021. Available: https://apps.who. int/iris/bitstream/handle/10665/339629/Policy-brief-39-1997-8073eng.pdf

17 Rayner C, Lokugamage AU, Molokhia M. Covid-19: prolonged and relapsing course of illness has implications for returning workers, 2020. Available: https://blogs.bmj.com/bmj/2020/06/23/covid-19prolonged-and-relapsing-course-of-illness-has-implications-forreturning-workers/

18 Pantelic M, Alwan N. The stigma is real for people living with long covid. thebmiopinion 2021.

19 Power K. The COVID-19 pandemic has increased the care burden of women and families. Sustainability: Science, Practice, and Policy 2020;16:67-73.

20 Wenham C, Smith J, Morgan R, et al. COVID-19: the gendered impacts of the outbreak. Lancet 2020;395:846-8.

21 Torjesen I. Covid-19: middle aged women face greater risk of debilitating long term symptoms. BMJ 2021;372:n829.

22 Office for National Statistics. Prevalence of ongoing symptoms following coronavirus (COVID-19) infection in the UK: 4 November 2021. estimates of the prevalence of self-reported long COVID and associated activity limitation, using UK coronavirus (COVID-19) infection survey data, 2021. Available: https://www.ons.gov.uk/peop lepopulationandcommunity/healthandsocialcare/conditionsanddis eases/bulletins/prevalenceofongoingsymptomsfollowingcoronavi ruscovid19infectionintheuk/1july2021 [Accessed 1 Nov 2021].

23 Vahidy FS, Pan AP, Ahnstedt $\mathrm{H}$, et al. Sex differences in susceptibility, severity, and outcomes of coronavirus disease 2019: cross-sectional analysis from a diverse US metropolitan area. PLoS One 2021;16:e0245556.

24 Halpin SJ, Mclvor C, Whyatt G, et al. Postdischarge symptoms and rehabilitation needs in survivors of COVID-19 infection: a crosssectional evaluation. J Med Virol 2021;93:1013-22.

25 Williamson EJ, Walker AJ, Bhaskaran K, et al. Factors associated with COVID-19-related death using OpenSAFELY. Nature 2020;584:430-6.

26 De Lorenzo R, Conte C, Lanzani C, et al. Residual clinical damage after COVID-19: a retrospective and prospective observational cohort study. PLoS One 2020;15:e0239570.

27 Lokugamage A, Rayner C, Simpson F. We have heard your message about long covid and we will act, says WHO, 2020. Thebmjopinion. Available: https://blogs.bmj.com/bmj/2020/09/03/we-have-heardyour-message-about-long-covid-and-we-will-act-says-who/

28 The Lancet. Understanding long COVID: a modern medical challenge. Lancet 2021;398:725.

29 World Physiotherapy. World physiotherapy response to COVID-19. Safe rehabilitation approaches for people living with long COVID: physical activity and exercise, 2021. Available: https://world.physio/ sites/default/files/2021-07/Briefing-Paper-9-Long-Covid-FINALEnglish-202107.pdf

30 World Health Organization. Rehabilitation needs of people recovering from COVID-19, 2021. Available: https://www.who.int/ publications/m/item/WHO-2019-nCoV-Sci_Brief-Rehabilitation2021.1 [Accessed 31 Dec 2021].

31 World Health Organization. COVID-19 clinical management living guidance, 2021.

32 Altiery De Jesús VV, Alwan N, Callard F. Listening to Long COVID: Epistemic Injustice and COVID-19 morbidity. OSF Preprints, 2021 Available: https://osf.io/tfbnd/

33 Brown DA, O'Brien KK, Josh J, et al. Six lessons for COVID-19 rehabilitation from HIV rehabilitation. Phys Ther 2020;100:1906-9.

34 Gorna R, MacDermott N, Rayner C, et al. Long COVID guidelines need to reflect lived experience. Lancet 2021;397:455-7.

35 Greenhalgh T, Knight M, A'Court C, et al. Management of postacute covid-19 in primary care. BMJ 2020;370:m3026. 
36 Shah W, Hillman T, Playford ED, et al. Managing the long term effects of covid-19: summary of NICE, SIGN, and RCGP rapid guideline. BMJ 2021;372:n136.

37 Ziauddeen N, Gurdasani D, O'Hara ME. Characteristics of long COVID: findings from a social media survey. medRxiv 2021.

38 Brown DA, O'Brien KK. Conceptualising long COVID as an episodic health condition. BMJ Glob Health 2021;6.

39 O'Brien KK, Bayoumi AM, Strike C, et al. Exploring disability from the perspective of adults living with HIV/AIDS: development of a conceptual framework. Health Qual Life Outcomes 2008;6:76.

40 Solomon P, O'Brien KK, Nixon S, et al. Trajectories of episodic disability in people aging with HIV: a longitudinal qualitative study. $J$ Int Assoc Provid AIDS Care 2018;17:2325958218759210.

41 O'Brien KK, Davis AM, Strike C, et al. Putting episodic disability into context: a qualitative study exploring factors that influence disability experienced by adults living with HIV/AIDS. J Int AIDS Soc 2009;12:5.

42 Davis HE, Assaf GS, McCorkell L, et al. Characterizing long COVID in an international cohort: 7 months of symptoms and their impact. EClinicalMedicine 2021:38:101019.

43 O'Brien KK, Hanna S, Gardner S, et al. Validation of the episodic disability framework with adults living with HIV. Disabil Rehabil 2014;36:319-29.

44 O'Brien KK, Bergin C, Solomon P, et al. Cross-cultural applicability of the episodic disability framework with adults living with HIV in Ireland: a qualitative study. Disabil Rehabil 2021;43:229-40.

45 World Health Organization. World Health Organizations disability assessment schedule II (WHO-DAS-II) 2001.

46 Sivan M, Halpin S, Gee J, et al. Assessing long-term rehabilitation needs in COVID-19 survivors using a telephone screening tool (C19-YRS tool). ACNR 2020;19:14-17.

47 O'Brien KK, Bayoumi AM, King K, et al. Community engagement in health status instrument development: experience with the HIV disability questionnaire. Prog Community Health Partnersh 2014;8:549-59.

48 O'Brien KK, Bayoumi AM, Stratford P, et al. Which dimensions of disability does the HIV disability questionnaire (HDQ) measure? a factor analysis. Disabil Rehabil 2015;37:1193-201.

49 O'Brien KK, Solomon P, Bayoumi AM. Measuring disability experienced by adults living with HIV: assessing construct validity of the HIV disability questionnaire using confirmatory factor analysis. BMJ Open 2014;4:e005456

50 Brown DA, Simmons B, Boffito M, et al. Evaluation of the psychometric properties of the HIV disability questionnaire among adults living with HIV in the United Kingdom: a cross-sectional selfreport measurement study. PLoS One 2019;14:e0213222.

51 O'Brien KK, Bayoumi AM, Bereket T, et al. Sensibility assessment of the HIV disability questionnaire. Disabil Rehabil 2013;35:566-77.

52 O'Brien KK, Solomon P, Bergin C, et al. Reliability and validity of a new HIV-specific questionnaire with adults living with HIV in Canada and ireland: the HIV disability questionnaire (HDQ). Health Qual Life Outcomes 2015;13:124.

53 O'Brien KK, Kietrys D, Galantino ML, et al. Reliability and validity of the HIV disability questionnaire (HDQ) with adults living with HIV in the United States. J Int Assoc Provid AIDS Care 2019;18:23259582 19888461:232595821988846.

54 O'Brien KK, Dzingina M, Harding R, et al. Developing a shortform version of the HIV disability questionnaire (SF-HDQ) for use in clinical practice: a Rasch analysis. Health Qual Life Outcomes 2021;19:6.

55 O'Brien KK, Brown DA, Corbett C, et al. AIDSImpact special issue - broadening the lens: recommendations from rehabilitation in chronic disease to advance healthy ageing with HIV. AIDS Care 2020;32:65-73.

56 Solomon P, O'Brien KK, Baxter L, et al. Community involvement in development of evidence-informed recommendations for rehabilitation for older adults living with HIV. Prog Community Health Partnersh 2016;10:83-8.

57 COVID long Haulers support group Canada (Goulding), 2021. Available: https://covidlonghaulcanada.com/

58 Long COVID Support, 2021. Available: https://www.longcovid.org/

59 Long COVID Physio. Available: https://longcovid.physio/ https:// www.physio-pedia.com/Long_COVID2021

60 Patient-Led Research Collaborative, 2021. Available: https://patientr esearchcovid19.com/

61 Long COVID Ireland. Available: https://www.longcovidireland.org/ [Accessed 31 Dec 2021]

62 O'Brien KK, Solomon P, Worthington C, et al. Considerations for conducting web-based survey research with people living with human immunodeficiency virus using a community-based participatory approach. J Med Internet Res 2014;16:e81.
63 Dillman DA. Mail and Internet surveys: the tailored design method-2007 update with new Internet, visual, and mixed-mode guide. John Wiley \& Sons, 2011

64 EuroQuol. EQ-5D, 2021. Available: https://euroqol.org/eq-5dinstruments/ [Accessed 12 Nov 2021].

65 Mundt JC, Marks IM, Shear MK, et al. The work and social adjustment scale: a simple measure of impairment in functioning. $\mathrm{Br}$ $J$ Psychiatry 2002;180:461-4.

66 Qualtrics [program]. Provo, Utah, USA, 2017.

67 O'Connor RJ, Preston N, Parkin A. The COVID-19 Yorkshire rehabilitation scale (C19-YRS): application and psychometric analysis in a post-COVID-19 syndrome cohort. J Med Virol 2021.

68 Garrigues E, Janvier P, Kherabi Y, et al. Post-discharge persistent symptoms and health-related quality of life after hospitalization for COVID-19. J Infect 2020;81:e4-6.

69 Hsieh H-F, Shannon SE. Three approaches to qualitative content analysis. Qual Health Res 2005;15:1277-88.

70 Flicker S, Nixon SA. The DEPICT model for participatory qualitative health promotion research analysis piloted in Canada, Zambia and South Africa. Health Promot Int 2015;30:616-24.

71 Dierckx de Casterlé B, Gastmans C, Bryon E, et al. QUAGOL: a guide for qualitative data analysis. Int J Nurs Stud 2012;49:360-71.

72 NVivo qualitative data analysis software. Version 11. [program] 2017.

73 Tennant A, Conaghan PG. The Rasch measurement model in rheumatology: what is it and why use it? When should it be applied, and what should one look for in a Rasch paper? Arthritis Rheum 2007:57:1358-62.

74 Tennant A, Penta M, Tesio L, et al. Assessing and adjusting for cross-cultural validity of impairment and activity limitation scales through differential item functioning within the framework of the Rasch model: the PRO-ESOR project. Med Care 2004;42:I37-48.

75 Rusch T, Lowry PB, Mair P, et al. Breaking free from the limitations of classical test theory: developing and measuring information systems scales using item response theory. Inf Manage 2017;54:189-203.

76 Cappelleri JC, Jason Lundy J, Hays RD. Overview of classical test theory and item response theory for the quantitative assessment of items in developing patient-reported outcomes measures. Clin Ther 2014;36:648-62.

77 Nunnally JC, Bernstein IH. Psychometric theory. 3rd edn. New York: McGraw-Hill, 1978

78 Tennant A, McKenna SP, Hagell P. Application of Rasch analysis in the development and application of quality of life instruments. Value Health 2004;7(Suppl 1):S22-6.

79 Brazier JE, Rowen D, Mavranezouli I, et al. Developing and testing methods for deriving preference-based measures of health from condition-specific measures (and other patient-based measures of outcome). Health Technol Assess 2012;16:1-114.

80 Bland JM, Altman DG. Multiple significance tests: the Bonferroni method. BMJ 1995;310:170.

81 Tennant A, Pallant JF. Unidimensionality matters. Rasch Meas Trans 2006;20:1048-51.

82 Perruccio AV, Stefan Lohmander L, Canizares M, et al. The development of a short measure of physical function for knee OA KOOS-Physical Function Shortform (KOOS-PS) - an OARSI/ OMERACT initiative. Osteoarthritis Cartilage 2008;16:542-50.

83 Aaronson N, Alonso J, Burnam A, et al. Assessing health status and quality-of-life instruments: attributes and review criteria. Qual Life Res 2002;11:193-205.

84 Landis JR, Koch GG. The measurement of observer agreement for categorical data. Biometrics 1977;33:159-74.

85 Terwee CB, Bot SDM, de Boer MR, et al. Quality criteria were proposed for measurement properties of health status questionnaires. J Clin Epidemiol 2007;60:34-42.

86 Hatcher L. A step-by-step approach to using SAS for factor analysis and structural equation modeling. Cary, NC, USA: SAS Institute Inc, 1994.

87 Schumacker RE, Lomax RG. A Beginner's Guide to Structural Equation Modeling. 2nd edn. Lawrence Erlbaum Associates, Inc, 2004.

88 Kline RB. Principles and practice of structural equation modeling. 3rd edn. New York, NY: The Guilford Press, 2011.

89 LT H, Bentler PM. Fit indices in covariance structure modeling: sensitivity to underparameterized model misspecification. Psychol Methods 1998;3:424-53.

90 LT H, Bentler PM. Cutoff criteria for fit indexes in covariance structure analysis: conventional criteria versus new alternatives. Structural Equation Modeling 1999;6:1-55.

91 Brown TA. Confirmatory factor analysis for applied research. New York, NY: The Guilford Press, 2006. 
92 SAS Computer Software 9.3 [program], 2011.

93 RUMM2030 [program]. Duncraig, Western Australia, 2018.

94 Mplus Version 8.2 [program]. Los Angeles, CA, 2018.

95 Hennink MM, Kaiser BN, Marconi VC. Code saturation versus meaning saturation: how many interviews are enough? Qual Health Res 2017;27:591-608.

96 Sample Size and Item Parameter Estimation Precision When Utilizing the One-Parameter "Rasch" Model Annual Meeting of the Mid-Western Educational Research Association 2015; Evanston, Illinois.

97 Canada-International HIV and rehabilitation research collaborative (CIHRRC), 2021. Available: http://cihrrc.ca

98 O'Brien KK, Solomon P, Ibáñez-Carrasco F, et al. Evolution of an international research collaborative in HIV and rehabilitation: community engaged process, lessons learned, and recommendations. Prog Community Health Partnersh 2018;12:395-408.

99 Carson G, Long Covid Forum Group. Research priorities for long Covid: refined through an international multi-stakeholder forum. BMC Med 2021;19:84.

100 Negrini S, Mills J-A, Arienti C, et al. "Rehabilitation research framework for patients with COVID-19" defined by cochrane rehabilitation and the World Health Organization rehabilitation programme. Arch Phys Med Rehabil 2021;102:1424-30.

101 Citrix. ShareFile, (02021 Citrix Systems, Inc, 2021. Available: http:// www.sharefile.com/ 